\title{
Criticality and network structure drive emergent oscillations in a stochastic whole-brain model
}

\author{
Giacomo Barzon ${ }^{1,2}$, Giorgio Nicoletti ${ }^{1}$, Benedetta Mariani ${ }^{1,2}$, \\ Marco Formentin ${ }^{2,3}$, Samir Suweis ${ }^{1,2}$ \\ ${ }^{1}$ Laboratory of Interdisciplinary Physics, Department of Physics and Astronomy \\ "G. Galilei", University of Padova, Padova, Italy \\ ${ }^{2}$ Padova Neuroscience Center, University of Padova, Padova, Italy \\ ${ }^{3}$ Department of Mathematics "Tullio Levi-Civita", University of Padova, Padova, \\ Italy \\ E-mail: giacomo.barzon.1@phd.unipd.it, samir.suweis@unipd.it
}

\begin{abstract}
Understanding the relation between the structure of brain networks and its functions is a fundamental open question. Simple models of neural activity based on real anatomical networks have proven effective in describing features of whole-brain spontaneous activity when tuned at their critical point. In this work, we show that indeed structural networks are a crucial ingredient in the emergence of synchronized oscillations in a whole-brain stochastic model at criticality. We study such model in the mean-field limit, providing an analytical understanding of the associated first-order phase transition, arising from the presence of a bistable region in the parameters space. Then, we derive the power spectrum in the linear noise approximation and we show that, in the mean-field limit, no global oscillations emerge. Finally, by adding back an underlying brain network structure with homeostatic normalization, we numerically show how the bi-stability region is disrupted and concomitantly a synchronized phase with maximal dynamic range is observed. Hence, both the structure of brain networks and criticality are fundamental in driving the collective coordinated responses and maximal sensitivity of whole-brain stochastic models.
\end{abstract}

Keywords: brain criticality, synchronized neural oscillations, brain networks, stochastic model, mean field 
Brain network structure, criticality and the emergence of synchronized oscillations 2

\section{Introduction}

The human brain constitutes an impressively complex system, spanning several spatial scales of organizations from microcircuits to whole-brain networks. The comprehensive map of neural connections is referred as "connectome" [1]. However, it is typically unfeasible to reconstruct connectomes at the neuronal scale, and often one relies on an anatomical connectivity at coarser spatial scales. In humans, such brain structural networks are typically assessed with diffusion tensor/spectrum imaging techniques, which quantify the white matter pathways between mesoscopic brain regions [2, 3].

These complex interconnections act as a backbone on top of which the neurophysiological dynamics occurs. One way to measure such neural activity is through functional magnetic resonance imaging (fMRI). Correlations in the fMRI signals of spontaneous activity during rest have been repeatedly observed [4], yielding detailed maps of complex emergent patterns of coherent brain activities, called resting state (functional) networks (RSN) [5]. Such patterns, that are consistent among healthy individuals [6], are specifically associated with neuronal systems responsible for sensory, cognitive, and behavioral functions [7, 8].

An hypothesis that is increasingly being considered in light of growing experimental [9, 10] and theoretical [11, 12, 13] results, is that collective emergent patters are signatures of brain self-organization to a critical point [14, 15], i.e., the brain dynamics may be poised at the edge of a phase transition [16]. The main evidences for this hypothesis are the presence of scale-free neural avalanches [17, 18] and cluster size distributions [19, 20], long-range temporal [21] and spatial [22, 23] correlations during spontaneous brain activity - exemplary properties of a system near its critical point. Some works have also suggested that such phenomenology is compatible with systems between an asynchronous phase and a synchronous one, with emerging oscillations [10, 24, 25]. In all these studies the role of the network structure in driving such emerging patterns - e.g., syncronized oscillations or optimal information processing - is often missing.

In fact, the emerging collective dynamics in the brain is shaped both by the underlying connectome and by the neural population activities [26, 27, 28]. Despite a direct correspondence between structural and functional networks, to what extent structure does determine the neural dynamics and its critical signatures has still to be clarified [29, 30]. Computational models may be the key to bridging this gap [31]. To this end, biophysically inspired models of neural dynamics are typically built on top of empirically derived structural networks, with the aim of reconciling functional behavior.

In particular, a stochastic version of the Greenberg \& Hastings cellular 
Brain network structure, criticality and the emergence of synchronized oscillations 3 automaton [32] - which is one of the simplest model to describe the neural dynamics - running over a human connectome of $N=998$ cortical regions [33] was shown to match some features of whole-brain activity when tuned to the critical point [19, 22]. Indeed, the model undergoes a critical transition as a function of an external parameter, that governs the level of induced excitatory activation by neighboring neurons.

Recent numerical studies highlighted how the topological details of the underlying network are crucial in shaping the type of the transition. Indeed, for densely connected networks the system exhibits a first order discontinuous transition [34, 35], with the activity of the network jumping discontinuously between a quiescent and an overactive phase, while for sparse networks the transition disappears [34, 36], suggesting that brain dynamics in individuals affected by stroke will not be critical [37. Nevertheless, an analytical understanding of such critical transition, and thus the disentangling of the role of the connectome structure, criticality and emergent properties is still missing.

In this work, we develop a stochastic continuous time formulation of the Greenberg \& Hastings model via a master equation approach. We show analytically how in the mean-field limit two stable equilibria emerge, together with a bistable region of the parameter space where these two equilibria coexist, allowing a deeper understanding of the nature of the model critical transition. Then, we derive the power spectrum of the oscillations and we show that it does not display any characteristic peak, i.e., we do not observe synchronized neural activity. However, when we go beyond the mean-field by adding a network connecting the different brain regions, we find that synchronized sustained oscillations emerge and that the bistable region shrinks and eventually disappears. Moreover, we show that the dynamic range [38] is maximal at the critical point of the model, hinting at the optimal information processing that typically emerges at criticality.

Overall, our results shed light on the role of the underlying network structure in the emergent collective patterns observed in the brain, as well as explaining the mechanisms behind the phase diagram of the Greenberg \& Hastings model reported in previous works [19, 22, 34, 35, 36, 37].

\section{Methods}

\subsection{Whole-brain stochastic continuous time model}

Here, we develop a continuous time formulation of the whole brain stochastic model introduced by Haimovici et al. [22] to describe the dynamics of the human brain at a mesoscopic scale. Such a model is a variation of the Greenberg \& Hastings cellular automaton [32] - GH from now on -, originally designed to study excitable media. 
Brain network structure, criticality and the emergence of synchronized oscillations 4

Briefly, each node in the system belongs to one of three states: quiescient $Q$, excited $E$, or refractory $R$. The original dynamics of the GH automaton is modified in such a way that the states undergo the following stochastic transitions:

$$
\begin{cases}Q \rightarrow E & \text { if } \sum_{j} W_{i j} s_{j}(t)>T \text { or with prob. } r_{1} \\ E \rightarrow R & \text { with prob. } 1 \\ R \rightarrow Q & \text { with prob. } r_{2}\end{cases}
$$

where $s_{j}(t) \in\{0,1\}$ is the state of node $j$ at a certain time step $t$ - set to 1 if the node is in the $E$ state, and 0 otherwise -, $W_{i j}$ is the weighted connectivity matrix of the underlying network, $r_{1}$ is the probability of self-activation and $r_{2}$ is the probability of recovery from the refractory state. In particular, $T$ is a threshold which governs the induced activation due to interaction with neighboring nodes, which acts as a control parameter of the model.

Hence, in this model a neuron may either be activated if the weighted combined activity of neighboring neurons exceeds a threshold $T$, or it may self-activate with a probability $r_{1}$ that encodes, e.g., external stimuli or unobserved pathways. After activation, neurons switch to a refractory state with unitary probability and cannot activate again. Finally, the escape from the refractory state happens with probability $r_{2}$. In this formulation, the state of the system evolves in discrete time steps and is updated synchronously.

By varying $T$, the model undergoes a phase transition [22]. In particular, the order parameters used to characterize the transition were the mean network activity, i.e., the fraction of active nodes, and the size of the clusters, i.e., sets of nodes structurally connected to each other and simultaneously active. For small values of $T$, the activity spread easily between neighboring nodes, even along weak connections. This leads to a regime of high and sustained activation, characterized by fast and temporally uncorrelated fluctuations and large clusters. We refer to this phase as "super-critical". On the other hand, for high values of $\mathrm{T}$, the activity is sustained only by few strong connections, resulting in a suppressed or "sub-critical" phase with regular, short propagating activity in which nodes fail to give rise to relevant clusters. At intermediate values of $\mathrm{T}$, a continuous transition connects the two regimes, resembling a second-order phase transition.

Notably, we include homeostatic plasticity in the model, implemented as a normalization of the incoming node's excitatory input. This addition minimizes both the variability of the critical points and, at the same time, it improves the correspondence between simulated neural patterns and experimental brain functional data [19].

We now develop a continuous time formulation of the model in order to study analytically its mean-field behavior in the large $N$ limit, together with its power 
Brain network structure, criticality and the emergence of synchronized oscillations 5

spectrum in the stochastic linearized regime. Given a network of $N$ units, we denote by $\sigma_{i}(t) \in\{E, R, Q\}, i=1, \ldots, N$, the state of the site $i$ at time $t$. The dynamics in (1) can be translated into the following continuous time evolution: for $h>0$ and each node $i$, the probability of having $\sigma_{i}(t+h)=E$ given that $\sigma_{i}(t)=Q$ is $r_{\text {act }}(i) h+o(h)$ where $r_{\text {act }}(i)$ is the rate of activation, defined as

$$
r_{a c t}(i)=r_{1}+\left(1-r_{1}\right) \Theta\left[\sum_{j} W_{i j} s_{j}-T\right]
$$

with $\Theta[\cdot]$ the Heaviside step function. Notice that $0 \leq r_{1} \leq 1$ by construction. In a similar manner the probability of jumping from state $E$ at time $t$ to state $R$ at time $t+h$ will be $h+o(h)$ and from $R$ to $Q$ will be $r_{2} h+o(h) \nsubseteq$.

The mean-field approximation of the model consists in assuming that the underlying graph is fully-connected with constant weights, i.e., $W_{i j}=c, \forall i, j$. In this way, considering also the homeostatic normalization [19], the weights of the structural matrix are simply $\widetilde{W}_{i j}=W_{i j} / \sum_{j} W_{i j}=1 / N$. Thus the activation of a node due to the neighboring nodes is simply given by the density of active nodes in the network, i.e., the argument inside $\Theta[\cdot]$ in (2) becomes

$$
\sum_{j} \widetilde{W}_{i j} s_{j}-T=\frac{n_{E}}{N}-T
$$

and it is independent of the particular node $i$, i.e., $r_{a c t}(i)=r_{a c t}$.

These transition rules induce a Markovian dynamics on $n_{E}, n_{R}, n_{Q}=N-$ $n_{E}-n_{Q}$, respectively the number of active, refractory and inactive nodes, with the following rates:

$$
\begin{array}{lll}
\left(n_{E}, n_{R}, n_{Q}\right) & \stackrel{n_{Q} r_{\mathrm{act}}}{\longrightarrow} & \left(n_{E}+1, n_{R}, n_{Q}-1\right) \\
\left(n_{E}, n_{R}, n_{Q}\right) & \stackrel{n_{E}}{\longrightarrow} & \left(n_{E}-1, n_{R}+1, n_{Q}\right) \\
\left(n_{E}, n_{R}, n_{Q}\right) & \stackrel{n_{R} r_{2}}{\longrightarrow} & \left(n_{E}, n_{R}-1, n_{Q}+1\right)
\end{array} .
$$

Then, from the reactions in (4), we can write

$$
\begin{aligned}
\dot{P}\left(n_{E}, n_{R}\right)= & P\left(n_{E}-1, n_{R}\right)\left[N-n_{E}-n_{R}+1\right] r_{a c t} \\
& +P\left(n_{E}+1, n_{R}-1\right)\left[n_{E}+1\right] \\
& +P\left(n_{E}, n_{R}+1\right)\left[n_{R}+1\right] r_{2} \\
& -P\left(n_{E}, n_{R}\right)\left[\left(N-n_{E}-n_{R}\right) r_{a c t}+n_{E}+n_{R} r_{2}\right]
\end{aligned}
$$

which is the master equation of our continuous time model.

$\ddagger$ We highlight that the parameters $r_{1}$ and $r_{2}$ that in the time discrete model were probabilities are now rates 
Brain network structure, criticality and the emergence of synchronized oscillations 6

\subsection{Equlibria and power spectrum}

In order to study analytically the dynamics given by the master equation (5), we perform its Kramers-Moyal expansion truncated at second order. In this way, we can derive the associated Fokker-Planck and Langevin equations [39]. The latter describes the stochastic evolution of the density of active $x=n_{E} / N$ and refractory $y=n_{R} / N$ nodes, which obeys

$$
\left[\begin{array}{l}
\dot{x} \\
\dot{y}
\end{array}\right]=\left[\begin{array}{l}
A_{1}(x, y) \\
A_{2}(x, y)
\end{array}\right]+\frac{1}{\sqrt{N}}\left[\begin{array}{ll}
B_{11}(x, y) & B_{12}(x, y) \\
B_{21}(x, y) & B_{22}(x, y)
\end{array}\right]^{1 / 2}\left[\begin{array}{l}
\xi_{1} \\
\xi_{2}
\end{array}\right]
$$

where $\boldsymbol{\xi}=\left[\xi_{1}, \xi_{2}\right]$ is an uncorrelated $2 \mathrm{~d}$ white Gaussian noise, i.e., such that $\xi_{i} \sim N(0,1)$ and $\left\langle\xi_{i}(t) \xi_{j}\left(t^{\prime}\right)\right\rangle=\delta_{i j} \delta\left(t-t^{\prime}\right), \boldsymbol{A}(x, y)$ is the deterministic drift term, and $\boldsymbol{B}(x, y)$ encloses the stochastic diffusive part (see Appendix A for the full derivation).

To analytically investigate the oscillatory dynamics of our model, from (6) we perform a Van Kampen expansion [39] by defining the local coordinates $\left(\zeta_{1}, \zeta_{2}\right)$ as

$$
\left\{\begin{array} { l } 
{ x ( t ) = x ^ { * } + \frac { \zeta _ { x } ( t ) } { \sqrt { N } } } \\
{ y ( t ) = y ^ { * } + \frac { \zeta _ { y } ( t ) } { \sqrt { N } } }
\end{array} \Rightarrow \left\{\begin{array}{l}
\zeta_{x}(t)=\sqrt{N}\left(x(t)-x^{*}\right) \\
\zeta_{y}(t)=\sqrt{N}\left(y(t)-y^{*}\right)
\end{array} .\right.\right.
$$

Then (see Appendix $\mathrm{C}$ for details) the power spectrum of the oscillations around a given equilibrium is given by

$$
S_{i}(\omega)=\left\langle\tilde{\zeta}_{i}(\omega) \tilde{\zeta}_{i}^{*}(\omega)\right\rangle=\left\langle\tilde{\zeta}_{i}(\omega) \tilde{\zeta}_{i}(-\omega)\right\rangle
$$

for $i=x, y$.

\section{Results}

\subsection{Existence of a bistable region}

In the limit of a large number of interacting units in the system, the effect of random fluctuations become negligible. In fact, in the thermodynamic limit $N \rightarrow \infty$ the time evolution of the densities described by (6) converge, over a finite time interval, to the solutions of the following system of differential equations

$$
\left\{\begin{array}{l}
\dot{x}=(1-x-y)\left[r_{1}+\left(1-r_{1}\right) \Theta(x-T)\right]-x \\
\dot{y}=x-r_{2} y
\end{array}\right.
$$

which deterministically describes the dynamics of the concentrations of active and refractory units. Although we cannot obtain the full analytical solution of (9), we can study the system's equilibria and their stability. Indeed, by varying the threshold 
Brain network structure, criticality and the emergence of synchronized oscillations 7

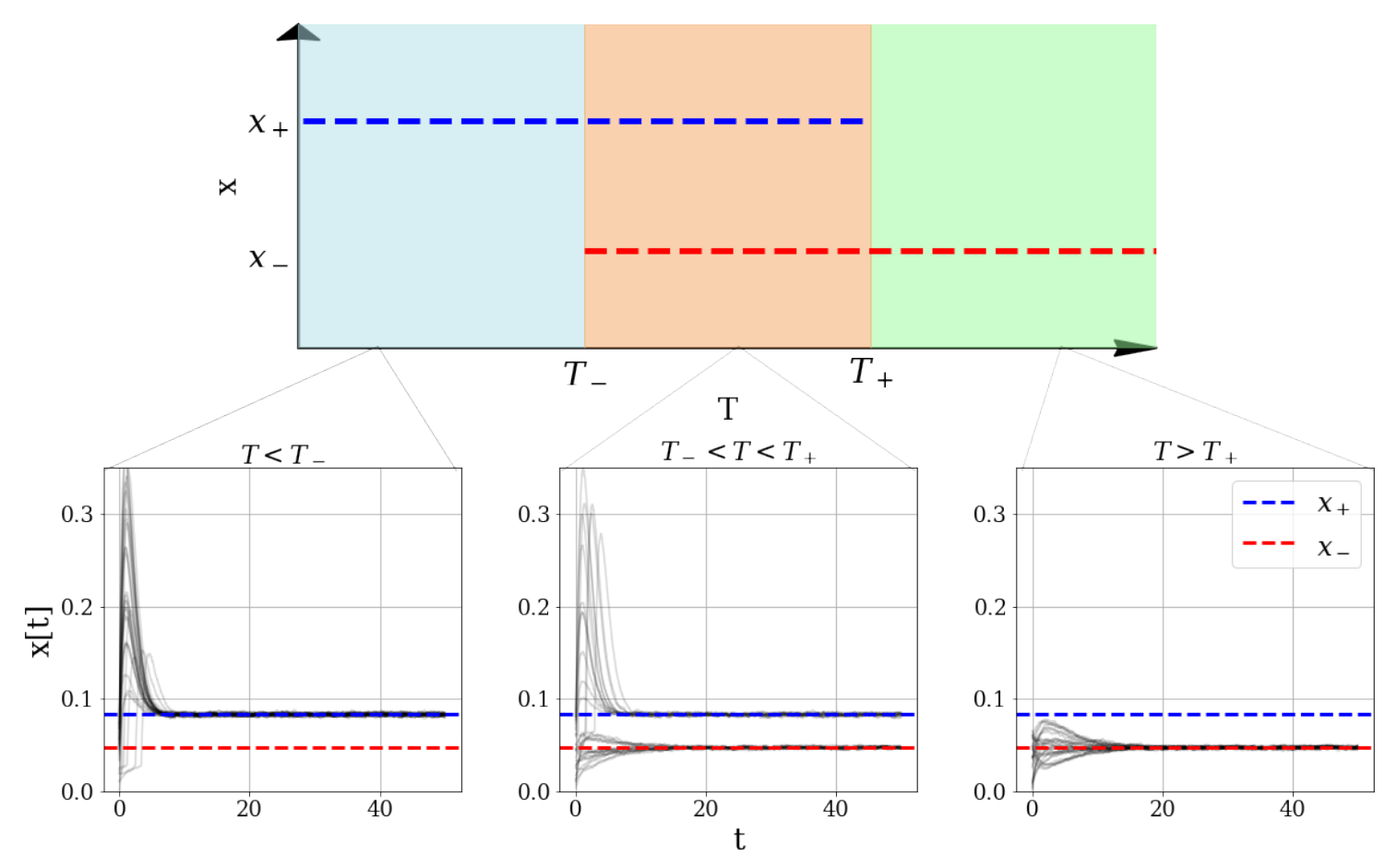

Figure 1: Diagram of equilibria in the model. Top panel: region of existence of superand sub-critical equilibria. As the control parameter T changes, we can identify three different regions: for low $\mathrm{T}$, only the supercritical equilibrium exists (blue region); for high $\mathrm{T}$, only the subcritical equilibrium exists (green region); for intermediate $\mathrm{T}$, there is the coexistence of the two equilibria (orange region). Bottom panel: examples of trajectories in the three regions. Model simulated with a fully-connected network of size $N=5 \cdot 10^{4}$ and with parameters $d t=0.001, r_{1}=0.1, r_{2}=0.1$. If not specified, these parameters are used throughout the work. Each plot shows 30 trajectories from a random initial configuration.

$T$ the dynamics switches between two different regimes based on the value of $\Theta[\cdot]$, as we see in figure 1. These two phases are characterized by high and low level of activity respectively. We call them super- and sub-critical phase.

In the super-critical phase $x>T$ and at stationarity, (9) leads to

$$
\left\{\begin{array}{l}
y_{+}=\frac{1}{2 r_{2}+1} \\
x_{+}=r_{2} y_{+}
\end{array}\right.
$$

so that in this regime the average activity $x_{+}$is independent from the rate of selfactivation $r_{1}$. This means that the spreading of the activity is completely driven by the interaction between active neighbors. For the equilibrium defined in 10 to 
Brain network structure, criticality and the emergence of synchronized oscillations 8

exists, we need

$$
T<\frac{r_{2}}{2 r_{2}+1}=: T_{+}
$$

so that the inequality $x>T$ is satisfied.

Likewise, in the sub-critical phase $x \leq T$ and at stationary, (9) leads to

$$
\left\{\begin{array}{l}
y_{-}=\frac{r_{1}}{r_{2}+\left(r_{2}+1\right) r_{1}} \\
x_{-}=r_{2} y_{-}
\end{array}\right.
$$

with the inequality $x \leq T$ leading to

$$
T \geq \frac{r_{1} r_{2}}{r_{2}+\left(r_{2}+1\right) r_{1}}=: T_{-} .
$$

As expected, from $(10)$ and $(12)$ we notice that $\forall r_{1}, r_{2}$ the fraction of active nodes $x_{+}$ in the supercritical phase is larger than the subcritical equilibrium $x_{-}$, since $r_{1} \leq 1$. Moreover, in the range of $T$ given by equations (11) and (13) for which such solutions exist, they are both stable equilibria, each one with its own basin of attraction (see Appendix B for the extensive analysis).

Crucially, and $\forall r_{1}, r_{2},(11)$ and (13) imply that $T_{-}<T_{+}$, thus three regions emerge in the parameter space spanned by $T$, as shown in Figure 1 . For $T \leq T_{-}$, the sub-critical equilibrium does not exist, hence we can only observe the super-critical equilibrium, whereas for $T>T_{+}$only the sub-critical equilibrium exists. In between these values, for $T_{-}<T \leq T_{+}$, the two equilibria coexist and we find a region of bistability.

\subsection{Power spectrum}

Neural activity typically exhibits a certain level of stochastic fluctuations, even when the brain is at rest [40]. In fact, a growing amount of evidence suggests that neural noise might enhance the signal processing capabilities of neurons [41, 42, 43]. To this end, we explore analytically the presence of oscillations in the model through the stochastic linearization given by a system size expansion [39], from which we obtain the temporal evolution of the fluctuations around the equilibria (see Appendix C). Indeed, this approach has proven to be effective in other neuronal models [28, 44, 45, 46], and leads to the power spectra

$$
S_{x}^{+}(\omega)=\frac{2 r_{2}\left[1+r_{2}+r_{2}^{2}+\omega^{2}\right]}{\left(1+2 r_{2}\right)\left[\left(1+2 r_{2}\right)^{2}+\left(2+r_{2}^{2}\right) \omega^{2}+\omega^{4}\right]}
$$

in the super-critical regime and

$$
S_{x}^{-}(\omega)=\frac{2 r_{1} r_{2}\left[r_{1}^{2}+r_{1} r_{2}+r_{2}^{2}+\omega^{2}\right]}{\left(r_{1}+r_{2}+r_{1} r_{2}\right)\left[\left(r_{1}+r_{2}+r_{1} r_{2}\right)^{2}+\left(1+r_{1}^{2}+r_{2}^{2}\right) \omega^{2}+\omega^{4}\right]}
$$


Brain network structure, criticality and the emergence of synchronized oscillations 9
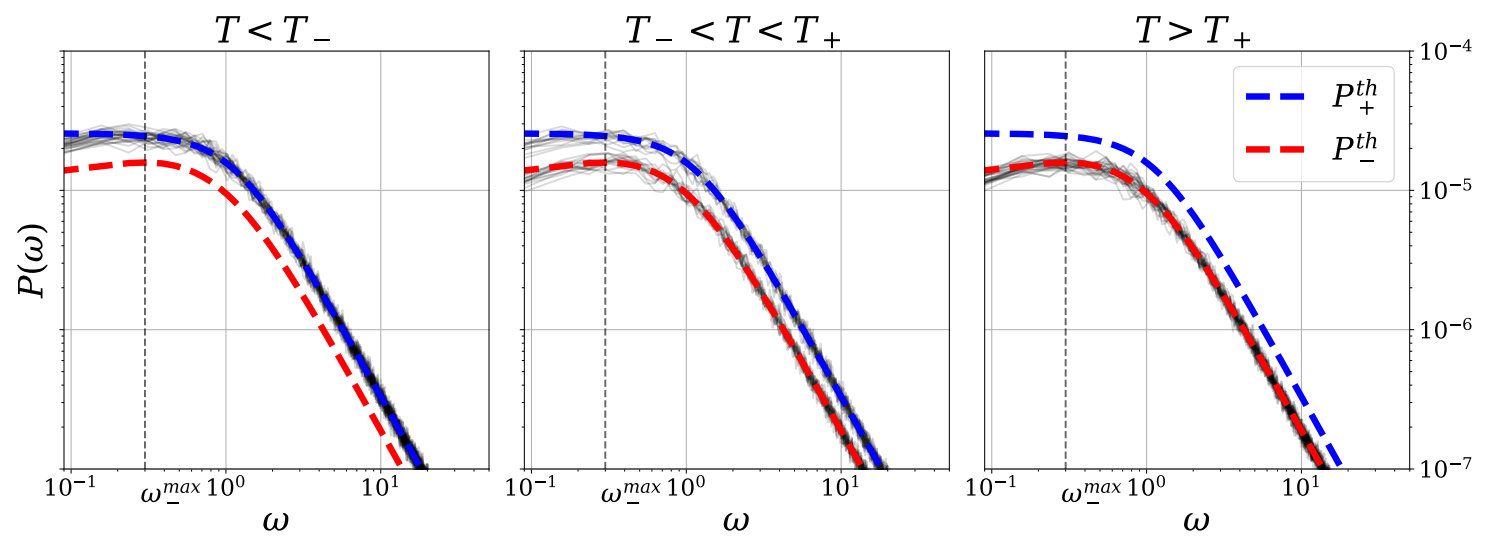

Figure 2: Theoretical expectation of the power spectrum are well-matched by simulated data from a fully-connected network at stationarity. The gray lines represent the power-spectrum obtained by simulating the continuous-time model for $10^{5}$ steps at stationarity in a network of $N=5 \cdot 10^{4}$ nodes and after an initial transient of $5 \cdot 10^{4}$ steps. In the bistable region, depending on which equilibria the dynamics settles, we can find both power spectra.

in the sub-critical regime. The power spectra obtained from the simulation of the model are perfectly matched by these theoretical expression (figure 2).

Equations (14) and (15) show that for low frequencies the power spectrum is flat in both regimes. On the other hand, in the large frequencies limit we find $S(\omega) \approx \omega^{-2}$, i.e., Brownian noise. Such scale-free behavior of the frequencies spectrum is found, for instance, in Local Field Potentials (LFPs), i.e., the electrical activity of the brain measured with single microelectrodes [47].

Notably, in the super-critical regime (15) does not display any peak, while a extremely small peak at

$$
\omega_{-}^{\max }=\left[\left(1+r_{1} r_{2}\right)\left(r_{1} r_{2}\right)^{1 / 2}-r_{1}^{2}-r_{2}^{2}-r_{1} r_{2}\right]^{1 / 2}
$$

emerges in the sub-critical phase. These results suggests that the mean-field limit of the model does not display any collective oscillations.

\subsection{Finite size effects}

In order to assess the effects of finite sizes on the region of bistability, we track the average activity $\bar{x}$ as an order parameter following the same approach used in [35]. The simulation starts at $T_{0}$ from a random initial configuration and after a given number of steps we increase the control parameter $T$ by a small $\Delta T$, without resetting the system states. Such procedure is repeated up to a final value $T_{F}$. Then, the same procedure is repeated by starting from $T_{F}$ and decreasing $T$ up to $T_{0}$. 
Brain network structure, criticality and the emergence of synchronized oscillations10
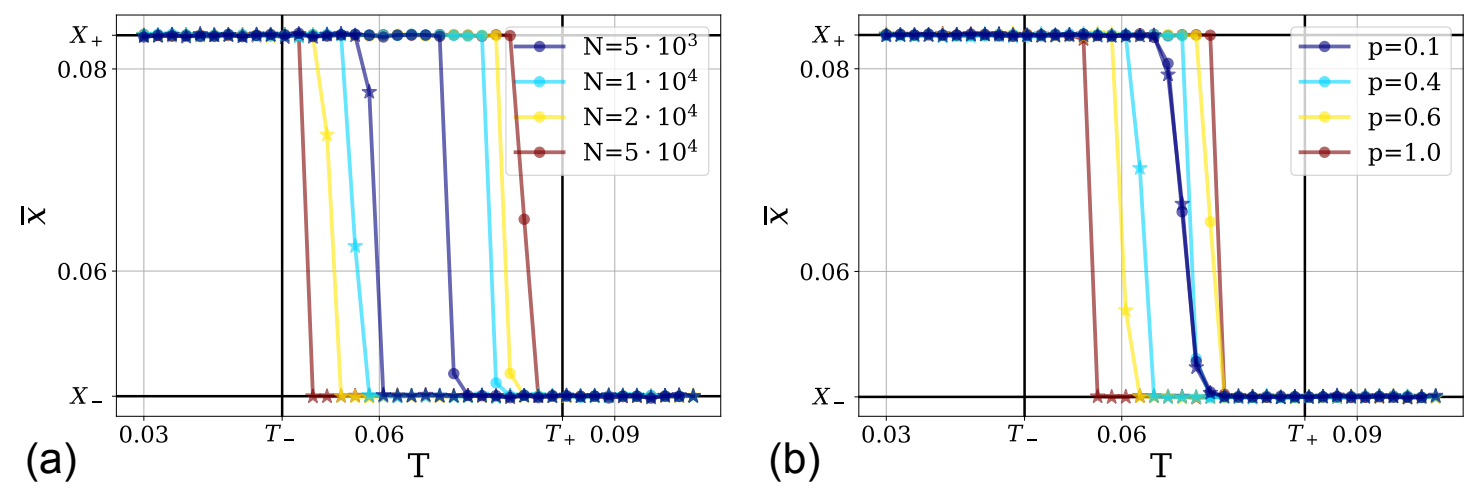

Figure 3: Trend of the average activity $\bar{x}$ as a function of the control parameter $\mathrm{T}$ for different topologies. Starting from $T_{0}=0.03$, $\mathrm{T}$ was slowly increased by $\Delta T=0.002$ every $10^{5}$ steps up to $T_{F}=0.10$, then it is decreased back to $T_{0}$ in the same way. (a) Results obtained with fully-connected topologies of different sizes $N$. (b) Results obtained with Erdős-Rényi random networks $\left(N=10^{4}\right)$ of different probabilities $p$ of having an edge between nodes.

In figure 3(a) we plot the behavior of $\bar{x}$ at different steps of this procedure for fully-connected topologies with different sizes. In the super- and sub-critical region $\bar{x}$ is in accordance with the theoretical predictions $(10)$ and $(12)$. We recover the discontinuous transition and the hysteresis cycle previously shown as well [34, 35]. Perhaps unsurprisingly, for small network sizes the transitions do not precisely match the expected values of $T_{+}$and $T_{-}$given in (11) and (13) - indeed, the bistable region shrinks as the size of the network is reduced.

\subsection{Effects of the network topology}

Until now, we have focused on the mean-field limit, which corresponds to a fullyconnected topology, and under the assumption of constant weights. However, the brain architecture is characterized by a sparse connectivity and brain networks display a non-trivial topology with small-world properties and community structures, both at micro and macro scale [33, 48]. Moreover, the strength of the interaction between different brain regions is highly heterogeneous, and typically follows a scalefree distribution [33, 48]. Hence, the hypothesis of constant weights is also not fulfilled, although eased by the homeostatic normalization [19]. In this section, we relax both these assumptions at the price of analytical tractability, showing how the network structure and criticality are both fundamental in shaping non-trivial behaviors that are not present in the mean-field limit.

As we have already mentioned, the effects of the underlying topology in shaping 
Brain network structure, criticality and the emergence of synchronized oscillations11
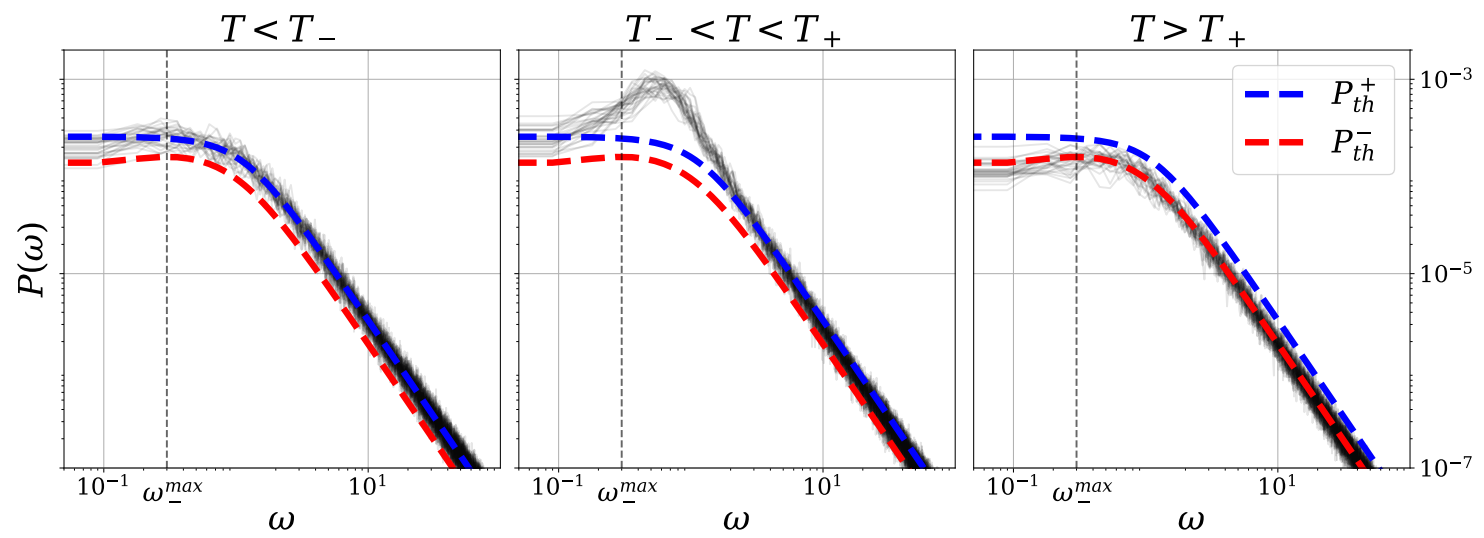

Figure 4: Dynamics of the model over an empirical connectome shows emergent collective oscillations. Simulation performed as in figure 2 with the connectome from [33].

the transition was extensively studied in the discrete time model [19, 34, 35, 36, 37]. To check if the same relation holds with our continuous time formulation as well, we simulate the dynamics over Erdős-Rényi networks [49].

We repeat the procedure described in section 3.3 at fixed network size but for different wiring probabilities. We find that, as we lower the connectivity, the bistable regions shrinks until it disappears, giving rise to a continuous transition (figure 3(b)). This behavior, which is deeply different from the one expected in the mean-field approximation, is consistent with what was found in the discrete time case.

Finally, we consider an empirical connectome of the human cerebral cortex with $N=998$ regions [33]. Numerical simulations show that our analytical results are still predictive in the limit of small and large values of $T$, whereas for intermediate values of the control parameter the average activity is no longer bounded to the two equilibria, but it rather jumps continuously from one to the other. This phenomenon gives rise to synchronized collective oscillations. Indeed, in the bistable region the power spectrum now displays a significant peak, see figure 4, which is not present in the mean-field limit.

\subsection{Dynamic range is maximized at criticality}

The rate of self-activation can be seen as an external stimulus that triggers the activation of the neurons. Indeed, the larger the stimulus, the higher the overall activity of the network. However, the shape of the response strictly depends on the threshold level. In particular, the control parameter affects the range of external stimuli that the system encodes in perceptible variations of the response. Such 
Brain network structure, criticality and the emergence of synchronized oscillations12

property is quantified in the dynamic range [50]

$$
\Delta=10 \log \left(\frac{r_{1}^{\text {high }}}{r_{1}^{\text {low }}}\right)
$$

that is the ratio between the largest $r_{1}^{\text {high }}$ and the smallest $r_{1}^{\text {low }}$ stimuli that results in relevant variations of the response of the system [38].

The range $\left[r_{1}^{\text {low }}, r_{1}^{\text {high }}\right]$ is found from the corresponding response interval $\left[\bar{x}_{\text {low }}, \bar{x}_{\text {high }}\right]$, where

$$
\bar{x}_{k}=\bar{x}_{\text {min }}+k\left(\bar{x}_{\text {max }}-\bar{x}_{\text {min }}\right)
$$

Notice that the choice of $\left[\bar{x}_{l o w}, \bar{x}_{h i g h}\right]$ is arbitrary. Typically, it is chosen in order to discard stimuli that are too weak or too close to saturation. Following [38], we define the range that produce respectively $10 \%$ and $90 \%$ of the total response curve. This specific choice is standard in the literature and does not affect qualitatively our results.

As we can see in figure 5, the dynamic range is maximized at the critical point of the model, that we identified in the peak of the average size of the second largest cluster as done in the original work [22]. Indeed, for low values of $T$ the dynamics is mostly governed by the interaction among neighbors, so even a weak stimulus leads to a high activity - meaning a suppressed dynamic range. The dynamic range is small even for high values of $T$, because activity in the network is triggered only with very high stimulus rates. Thus we have a picture where in the super-critical regime the dynamic range increases monotonically, while in the sub-critical phase it decreases.

\section{Discussion}

Models of large-scale neuronal dynamics are fundamental in explaining and predicting neuronal activity at the macroscopic scale [31]. Such models, that describe the collective behavior of populations of neurons, are inspired from biophysics and replicate observed phenomena of brain dynamics, e.g., scale-free avalanches [10, 17, 18], long-range correlations [21, 22, 23], synchronized oscillations [26, 27, 28]. However, the collective dynamics is crucially determined by both the dynamical rules chosen to model the inter-neuronal activations and by the geometry of their connections [35]. Disentangling the contributions of these two aspects is necessary to obtain a more clear and explicit understanding of the fundamental mechanisms behind the emergent patterns observed in the brain.

Driven by these considerations, in our work we have developed a continuous time version of a whole-brain stochastic model [22] and we have studied the nature of the associated critical transition. Even though in the last years several works has 
Brain network structure, criticality and the emergence of synchronized oscillations13
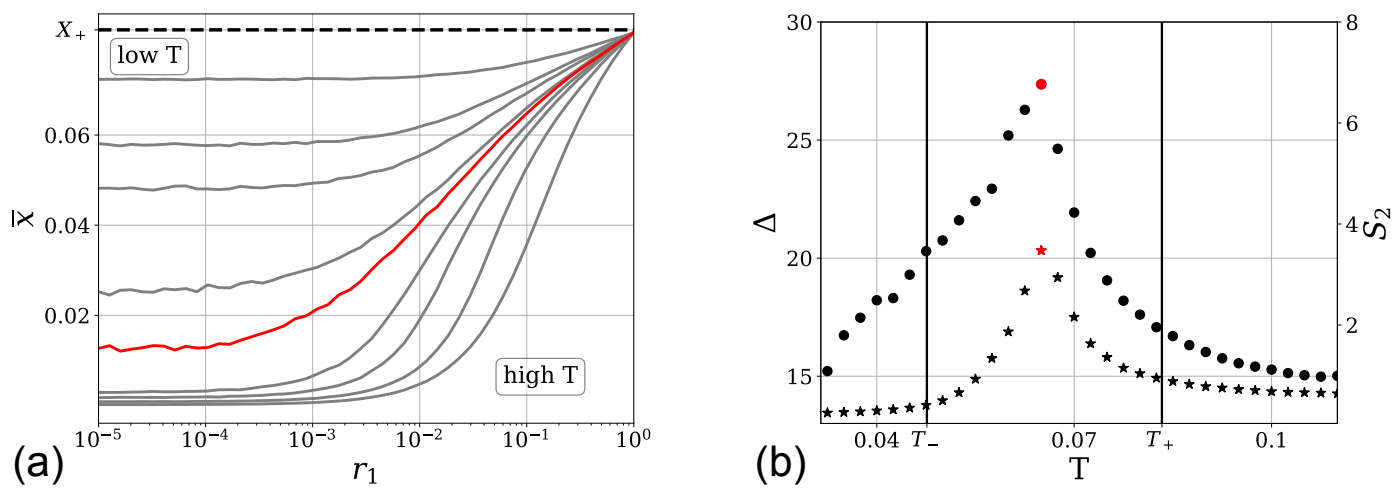

Figure 5: Dynamic range is maximal at criticality. (a) Response of the model, i.e. average activity $\bar{x}$, as a function of the rate of external stimuli $r_{1}$ for some values of the control parameter T. Each curve corresponds to the average over 100 trajectories. (b) Dynamic range is maximized at the critical value of $\mathrm{T}$, identified in the peak of second cluster size $S_{2}$ (starred points).

discussed the effect of the topology in shaping the transition by means of in-silico [34, 35, 36] and empirical connectomes [19, 37], to our current knowledge this is the first attempt to investigate the nature of the critical transition in this model from an analytical perspective.

Yet, the bistable region found in the mean-field limit lacks any sign of synchronized oscillations. It is only by introducing an empirical connectome that this bistability is disrupted, leading to the critical transition firstly shown in [22], and synchronized oscillations emerge at criticality. Therefore, both criticality and connectome structure play a fundamental role in driving the collective behavior of neurons and achieving optimal sensitivity when tuned at the critical point. Our findings are a further contribution into the still puzzling "critical brain hypothesis" [51].

The small size of the empirical connectome considered here may be a limitation to these investigations, since finite size corrections may be hiding criticality or rare region effects [52]. Notably, in [53] a similar modification of the discrete time Greenberg \& Hastings model run on a very large-scale connectome of almost $10^{6}$ nodes displays semicritical behaviors consistent with a Griffith phase in a certain range of the control parameter. The use of synthetic connectomes overcomes the finite size issue, at the cost of relying on some subjective assumptions on the generated topologies.

Another biological model of neuronal dynamics that shows an optimal dynamic range at criticality was proposed in the seminal work by Kinouchi and Copelli [38]. The authors claimed it was the "first clear example of signal processing optimization 
Brain network structure, criticality and the emergence of synchronized oscillations14

at a phase transition, making use of a standard and easily measurable performance index", and further works was carried out with non-trivial topologies, such as random and scale-free graphs [54, 55]. The fact that we observe a similar phenomenology of the dynamical range in our model is far from trivial. In fact, the model proposed by Kinouchi \& Copelli does not display the same phenomenological richness, since the transition is always continuous independently of the network topology [35].

Crucially, here we are able to show that the network structure seems to play a fundamental role, that future works should investigate further by going beyond the simple mean-field approach. Therefore we believe that our work will serve as a baseline for future analytical efforts in explaining the nature of the observed transition under more relaxed assumptions, e.g., in presence of non trivial distribution of weights and different topologies, to further understand the influence of both in the emergence of critical features in the human brain. Possible approaches may include the use of heterogeneous mean-field methods as done in the study of epidemic spreading [56], or annealed network approximations [57]. 
Brain network structure, criticality and the emergence of synchronized oscillations15

\section{Appendix A. System size expansion in the mean-field approximation}

The master equation (5) can be reframed in terms of the density of active $x$ and refractory $y$ neurons. Since $\Delta x=1 / N, \Delta y=1 / N$, we can treat them as continuous variables in the limit of a large system, i.e., $N \rightarrow \infty$, thus $P(x, y)$ becomes differentiable. By taking the continuum limit of the master equation (5) and by expanding all the terms, i.e., the Kramers-Moyal expansion [39], up to the second order, we obtain the so-called Fokker-Plank equation for the probability density $p(x, y)$

$$
\begin{aligned}
\frac{\partial}{\partial t} p(x, y)= & -\frac{\partial}{\partial x}\left[A_{1}(x, y) p(x, y)\right]+\frac{1}{2 N} \frac{\partial^{2}}{\partial x^{2}}\left[B_{11}(x, y) p(x, y)\right]+ \\
& -\frac{\partial}{\partial y}\left[A_{2}(x, y) p(x, y)\right]+\frac{1}{2 N} \frac{\partial^{2}}{\partial y^{2}}\left[B_{22}(x, y) p(x, y)\right]+ \\
& +\frac{1}{2 N} \frac{\partial^{2}}{\partial x \partial y}\left[\left(B_{12}(x, y)+B_{21}(x, y)\right) p(x, y)\right]
\end{aligned}
$$

where the coefficients are

$$
\left\{\begin{array}{l}
A_{1}(x, y)=(1-x-y)\left[r_{1}+\left(1-r_{1}\right) \Theta(x-T)\right]-x \\
A_{2}(x, y)=x-r_{2} y \\
B_{11}(x, y)=(1-x-y)\left[r_{1}+\left(1-r_{1}\right) \Theta(x-T)\right]+x \\
B_{22}(x, y)=r_{2} y+x \\
B_{12}(x, y)=-x \\
B_{21}(x, y)=-x
\end{array}\right.
$$

and $P(x, y)=p(x, y) \Delta x \Delta y$. The Fokker-Planck equation is a deterministic differential equation describing how the probability distribution of states $p(x, y)$ evolves over time. Physically, it describes the evolution of an ensemble of systems: if we simulate a huge number of populations of neurons, all with the same parameters, they will have different evolutions due to random fluctuations, but the fraction of systems that have a density of states in $[x, x+d x ; y, y+d y]$ at time $\mathrm{t}$ will be given exactly by $p(x, y) d x d y$ (in the limit of an infinite ensemble). An equivalent description can be derived by instead following a single population of neurons. In this case, a change in population density $[d x, d y]$ under the effect of stochastic fluctuations $\xi$ is given by the associated Langevin equation (6] [39]. 
Brain network structure, criticality and the emergence of synchronized oscillations16

\section{Appendix B. Stability analysis of equilibria}

We further investigate the nature of the equilibria through linear stability analysis techniques [58]. Indeed (9) is a dynamical system of the type:

$$
\dot{z}=\boldsymbol{f}(\boldsymbol{z})
$$

with $\boldsymbol{z}=(x, y)$ a 2 -dimensional vector. The equilibria $\boldsymbol{z}^{*}$ of this system are the ones that satisfy $\boldsymbol{f}\left(\boldsymbol{z}^{*}\right)=0$. If we focus on the dynamics near the fixed points, we can perform a change of variables $x=x^{*}+\Delta x, y=y^{*}+\Delta y$. In the limit of small variations $|\Delta \boldsymbol{z}| \rightarrow 0$, meaning that we are considering states infinitesimally near the fixed points, B.1) can be Taylor-expanded as

$$
\begin{aligned}
\dot{\Delta} \boldsymbol{z} & =\boldsymbol{f}\left(\boldsymbol{z}^{*}\right)+\left.\frac{\partial \boldsymbol{f}}{\partial \boldsymbol{z}}\right|_{\boldsymbol{z}=\boldsymbol{z}^{*}} \Delta \boldsymbol{z}+\ldots \\
& =\boldsymbol{J}\left(\boldsymbol{z}^{*}\right) \Delta \boldsymbol{z}
\end{aligned}
$$

Thus the dynamics near the fixed points is governed, at the first order, only by the Jacobian matrix $\boldsymbol{J}$. In particular, the (real part of) the eigenvalues $\lambda$ of $\boldsymbol{J}$ can tell us information regarding the stability or instability. If $\max \operatorname{Re}(\lambda)>0$, the trajectories asymptotically diverge from the equilibria, otherwise for $\max \operatorname{Re}(\lambda)<0$ the trajectories converge to the fixed point, which is stable in this case.

In the super-critical phase the jacobian evaluated at $\left(x_{+}, y_{+}\right)$is

$$
J_{+}=\left[\begin{array}{cc}
-2 & -1 \\
1 & -r_{2}
\end{array}\right]
$$

The eigenvalues of $\mathrm{B} .3$ are

$$
\lambda_{+}=-\frac{2+r_{2} \pm \sqrt{r_{2}^{2}-4 r_{2}}}{2}
$$

The stability condition holds if $\operatorname{Re}\left(\lambda_{+}\right)<0$. We can distinguish two regimes: if $r_{2} \geq 4$ the eigenvalues are purely real, otherwise they have an imaginary part. In both cases the conditions is satisfied, thus the super-critical fixed point $\left(x_{+}, y_{+}\right)$is respectively a stable knot and stable focus (figure B1.

Instead, in the sub-critical phase the jacobian evaluated at $\left[x_{-}, y_{-}\right]$is

$$
J_{-}=\left[\begin{array}{cc}
-1-r_{1} & -r_{1} \\
1 & -r_{2}
\end{array}\right]
$$

whose eigenvalues are

$$
\lambda_{-}=\frac{-\left(1+r_{1}+r_{2}\right) \pm \sqrt{\left(1+r_{1}+r_{2}\right)^{2}-4\left(r_{1}+r_{2}+r_{1} r_{2}\right)}}{2}
$$


Brain network structure, criticality and the emergence of synchronized oscillations17

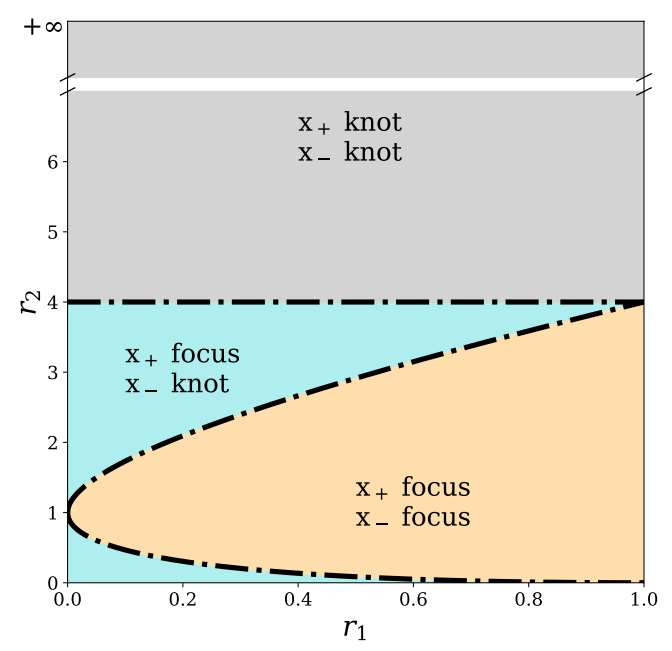

Figure B1: Type of fixed point in the parameter space $\left(r_{1}, r_{2}\right)$.

Also in this phase the stability condition is $\operatorname{Re}\left(\lambda_{-}\right)<0$ is satisfied $\forall r_{1}, r_{2}$ (since $r_{1} \geq 0$ and $r_{2} \geq 0$ ). We observe two different regimes by varying the parameters $r_{1}$ and $r_{2}$ : if $r_{1}-2 \sqrt{r_{1}}+1<r_{2}$ and $r_{1}+2 \sqrt{r_{1}}+1>r_{2}$ the eigenvalues have an imaginary part, while in the other case they are pure real. So in the first cases the fixed point is a stable focus, while it is a stable knot in the other case (figure B1).

\section{Appendix C. Power spectrum}

To study the effect of fluctuations around the equilibrium we make use of the van Kampen expansion [39]. First, we define here two local coordinates $\left(\zeta_{1}, \zeta_{2}\right)$

$$
\left\{\begin{array} { l } 
{ x ( t ) = x ^ { * } + \frac { \zeta _ { x } ( t ) } { \sqrt { N } } } \\
{ y ( t ) = y ^ { * } + \frac { \zeta _ { y } ( t ) } { \sqrt { N } } }
\end{array} \Rightarrow \left\{\begin{array}{l}
\zeta_{x}(t)=\sqrt{N}\left(x(t)-x^{*}\right) \\
\zeta_{y}(t)=\sqrt{N}\left(y(t)-y^{*}\right)
\end{array}\right.\right.
$$

In the Langevin equation, the stochastic fluctuations go as $1 / \sqrt{N}$, and so here we multiply by $\sqrt{N}$ to remove this size dependence.

Then, we rewrite the original equations in terms of $\left(\zeta_{x}, \zeta_{y}\right)$, keeping only the linear terms. For the deterministic part, this leaves only the jacobian evaluated at the equilibrium $J\left(x^{*}, y^{*}\right) \equiv J$. For the diffusion term we need to expand up to $1 / \sqrt{N}$ (so then we get order 1 after multiplying by $\sqrt{N}$ ). But this means that $b$ must be expanded to 0 -th order, otherwise we would have terms in $1 / N$, which become $1 / \sqrt{N}$ after multiplication, that are negligible in the thermodynamic limit. After this we 
Brain network structure, criticality and the emergence of synchronized oscillations18 arrive to:

$$
\left\{\begin{array}{l}
\dot{\zeta}_{x}=J_{11} \zeta_{x}+J_{12} \zeta_{y}+\eta_{x} \\
\dot{\zeta}_{y}=J_{21} \zeta_{x}+J_{22} \zeta_{y}+\eta_{y}
\end{array}\right.
$$

where

$$
\left(\begin{array}{c}
\eta_{x} \\
\eta_{y}
\end{array}\right)=\frac{1}{\sqrt{N}}\left(\begin{array}{c}
C_{1} \xi_{x}+C_{2} \xi_{y} \\
D_{1} \xi_{x}+D_{2} \xi_{y}
\end{array}\right)
$$

Since $\xi$ is Gaussian, also $\eta$ is Gaussian. However, since in $\eta$ we are summing over different components of $\xi, \eta_{1}$ and $\eta_{2}$ are not anymore uncorrelated:

$$
\left\langle\eta_{i}(t)\right\rangle=0 \quad\left\langle\eta_{i}(t) \eta_{j}\left(t^{\prime}\right)\right\rangle=\delta\left(t-t^{\prime}\right) B_{i j}
$$

where $B_{i j}=B_{i j}\left(x^{*}, y^{*}\right)$ is the diffusion matrix evaluated at equilibrium. To proceed, we move to Fourier space. Since the transformation is linear, it preserves the linearity of the equations:

$$
\left\{\begin{array}{l}
i \omega \tilde{\zeta}_{x}(\omega)=J_{11} \tilde{\zeta}_{x}+J_{12} \tilde{\zeta}_{y}+\tilde{\eta}_{x} \\
i \omega \tilde{\zeta}_{y}(\omega)=J_{21} \tilde{\zeta}_{x}+J_{22} \tilde{\zeta}_{y}+\tilde{\eta}_{y}
\end{array}\right.
$$

And the statistics of $\eta$ remain the same:

$$
\left\langle\tilde{\eta}_{i}(\omega)\right\rangle=0 \quad\left\langle\tilde{\eta}_{i}(\omega) \tilde{\eta}_{j}\left(\omega^{\prime}\right)\right\rangle=\delta\left(\omega-\omega^{\prime}\right) B_{i j}
$$

The linear system (Eq. C.5) leads to:

$$
\left\{\begin{array}{l}
\tilde{\zeta}_{x}=\frac{\left(i \omega-J_{22}\right) \tilde{\eta}_{x}+J_{12} \tilde{\eta}_{y}}{-\omega^{2}-i \omega\left(J_{11}+J_{22}\right)+J_{11} J_{22}-J_{12} J_{21}} \\
\tilde{\zeta}_{y}=\frac{\left(i \omega-J_{11}\right) \tilde{\eta}_{y}+J_{21} \tilde{\eta}_{x}}{-\omega^{2}-i \omega\left(J_{11}+J_{22}\right)+J_{11} J_{22}-J_{12} J_{21}}
\end{array}\right.
$$

Then we can study the power spectrum

$$
S_{i}(\omega)=\left\langle\tilde{\zeta}_{i}(\omega) \tilde{\zeta}_{i}^{*}(\omega)\right\rangle=\left\langle\tilde{\zeta}_{i}(\omega) \tilde{\zeta}_{i}(-\omega)\right\rangle
$$

that for the oscillations $\zeta_{x}$ of the density of active neurons $x$ leads to

$$
S_{x}(\omega)=\frac{\alpha+\beta \omega^{2}}{\left[\left(\omega^{2}-\Omega_{0}^{2}\right)^{2}+\Gamma^{2} \omega^{2}\right]}
$$

where

$$
\left\{\begin{array}{l}
\alpha=B_{11} J_{22}^{2}-2 B_{12} J_{12} J_{22}+B_{22} J_{12}^{2} \\
\beta=B_{11} \\
\Omega_{0}^{2}=J_{11} J_{22}-J_{12} J_{21} \\
\Gamma^{2}=\left(J_{11}+J_{22}\right)^{2}
\end{array}\right.
$$




\section{References}

[1] Leergaard T B, Hilgetag C C and Sporns O 2012 Frontiers in neuroinformatics 614

[2] Le Bihan D, Mangin J F, Poupon C, Clark C A, Pappata S, Molko N and Chabriat H 2001 Journal of Magnetic Resonance Imaging: An Official Journal of the International Society for Magnetic Resonance in Medicine 13 534-546

[3] Wedeen V J, Hagmann P, Tseng W Y I, Reese T G and Weisskoff R M 2005 Magnetic resonance in medicine $\mathbf{5 4}$ 1377-1386

[4] Fox M D and Raichle M E 2007 Nature reviews neuroscience 8 700-711

[5] Beckmann C F, DeLuca M, Devlin J T and Smith S M 2005 Philosophical Transactions of the Royal Society B: Biological Sciences 360 1001-1013

[6] Damoiseaux J S, Rombouts S, Barkhof F, Scheltens P, Stam C J, Smith S M and Beckmann C F 2006 Proceedings of the national academy of sciences 103 13848-13853

[7] Smith S M, Fox P T, Miller K L, Glahn D C, Fox P M, Mackay C E, Filippini N, Watkins K E, Toro R, Laird A R et al. 2009 Proceedings of the national academy of sciences 106 13040-13045

[8] Spadone S, Della Penna S, Sestieri C, Betti V, Tosoni A, Perrucci M G, Romani G L and Corbetta M 2015 Proceedings of the National Academy of Sciences 112 8112-8117

[9] Priesemann V, Levina A and Wilting J 2019 Assessing Criticality in Experiments (Cham: Springer International Publishing) pp 199-232 ISBN 978-3-030-20965-0

[10] Fontenele A J, de Vasconcelos N A P, Feliciano T, Aguiar L A A, Soares-Cunha C, Coimbra B, Dalla Porta L, Ribeiro S, Rodrigues A J a, Sousa N, Carelli P V and Copelli M 2019 Physical Review Letters 122(20) 208101

[11] Levina A, Herrmann J M and Geisel T 2009 Physical review letters 102118110

[12] Chialvo D R 2010 Nature physics 6 744-750

[13] Wilting J and Priesemann V 2019 Current Opinion in Neurobiology 58105 111 ISSN 0959-4388

[14] Hidalgo J, Grilli J, Suweis S, Munoz M A, Banavar J R and Maritan A 2014 Proceedings of the National Academy of Sciences 111 10095-10100

[15] Hesse J and Gross T 2014 Frontiers in systems neuroscience 8166

[16] Muñoz M A 2018 Review of Modern Physics 90(3) 031001

[17] Beggs J M and Plenz D 2003 Journal of neuroscience 23 11167-11177 
[18] Petermann T, Thiagarajan T C, Lebedev M A, Nicolelis M A L, Chialvo D R and Plenz D 2009 Proceedings of the National Academy of Sciences 106 1592115926 ISSN 0027-8424

[19] Rocha R P, Koçillari L, Suweis S, Corbetta M and Maritan A 2018 Scientific reports 8 1-15

[20] Korchinski D J, Orlandi J G, Son S W and Davidsen J 2021 Physical Review X 11021059

[21] Linkenkaer-Hansen K, Nikouline V V, Palva J M and Ilmoniemi R J 2001 Journal of Neuroscience 21 1370-1377

[22] Haimovici A, Tagliazucchi E, Balenzuela P and Chialvo D R 2013 Phys. Rev. Lett. 110(17) 178101

[23] Mariani B, Nicoletti G, Bisio M, Maschietto M, Oboe R, Leparulo A, Suweis S and Vassanelli S 2021 Frontiers in Systems Neuroscience 1589

[24] Markram H, Muller E, Ramaswamy S, Reimann M W, Abdellah M, Sanchez C A, Ailamaki A, Alonso-Nanclares L, Antille N, Arsever S et al. 2015 Cell 163 456-492

[25] Di Santo S, Villegas P, Burioni R and Muñoz M A 2018 Proceedings of the National Academy of Sciences 115 E1356-E1365

[26] Le Van Quyen M and Bragin A 2007 Trends in Neurosciences 30 365-373 ISSN 0166-2236

[27] Begleiter H and Porjesz B 2006 International Journal of Psychophysiology 60 162-171 ISSN 0167-8760 models and Theories of Brain Function with Special Emphasis on Cognitive Processing

[28] Apicella I, Busiello D, Scarpetta S and Suweis S 2021 Neurocomputing 461 716-726 ISSN 0925-2312

[29] Suárez L E, Markello R D, Betzel R F and Misic B 2020 Trends in Cognitive Sciences 24 302-315 ISSN 1364-6613

[30] Damoiseaux J S and Greicius M D 2009 Brain structure and function 213 525533

[31] Breakspear M 2017 Nature neuroscience 20 340-352

[32] Greenberg J M and Hastings S P 1978 SIAM Journal on Applied Mathematics $34515-523$

[33] Hagmann P, Cammoun L, Gigandet X, Meuli R, Honey C J, Wedeen V J and Sporns O 2008 PLoS Biol 6

[34] Zarepour M, Perotti J I, Billoni O V, Chialvo D R and Cannas S A 2019 Physical Review E 100052138 
[35] Diaz M M S, Trejo E J A, Martin D A, Cannas S A, Grigera T S and Chialvo D R 2021 Phys. Rev. E 104(6) 064309

[36] Haimovici A, Balenzuela P and Tagliazucchi E 2016 Brain connectivity 6 759771

[37] Rocha R P, Koçillari L, Suweis S, De Grazia M D F, de Schotten M T, Zorzi M and Corbetta M 2020 bioRxiv

[38] Kinouchi O and Copelli M 2006 Nature physics 2 348-351

[39] Van Kampen N G 1992 Stochastic processes in physics and chemistry vol 1 (Elsevier)

[40] Destexhe A and Rudolph-Lilith M 2012 Neuronal noise vol 8 (Springer Science \& Business Media)

[41] McDonnell M D and Ward L M 2011 Nature Reviews Neuroscience 12 415-425

[42] McDonnell M D and Abbott D 2009 PLoS computational biology 5 e1000348

[43] Guo D, Perc M, Liu T and Yao D 2018 EPL (Europhysics Letters) 12450001

[44] Ohira T and Cowan J D 1997 Stochastic neurodynamics and the system size expansion Mathematics of neural networks (Springer) pp 290-294

[45] Bressloff P C 2010 SIAM Journal on Applied Mathematics 70 1488-1521

[46] Fanelli D, Ginelli F, Livi R, Zagli N and Zankoc C 2017 Physical Review E 96 062313

[47] Milstein J, Mormann F, Fried I and Koch C 2009 PloS one 4 e4338

[48] Bullmore E and Sporns O 2009 Nature reviews neuroscience 10 186-198

[49] Erdös P and Rényi A 1959 Publicationes Mathematicae Debrecen 6 290-297

[50] Dayan P and Abbott L F 2001 Theoretical neuroscience: computational and mathematical modeling of neural systems (Computational Neuroscience Series)

[51] Beggs J M and Timme N 2012 Frontiers in physiology 3163

[52] Vojta T 2006 Journal of Physics A: Mathematical and General 39 R143

[53] Ódor G, Kelling J, Gastner M and Deco G 2021 Journal of Physics: Complexity

[54] Wu A C, Xu X J and Wang Y H 2007 Physical Review E 75032901

[55] Larremore D B, Shew W L and Restrepo J G 2011 Physical review letters 106 058101

[56] Pastor-Satorras R and Vespignani A 2001 Physical Review E 63066117

[57] Dorogovtsev S N, Goltsev A V and Mendes J F 2008 Reviews of Modern Physics 801275

[58] Sayama H 2020 Linear stability analysis of nonlinear dynamical systems 